Math. Model. Nat. Phenom.

Vol. 5, No. 3, 2010, pp. 40-62

DOI: $10.1051 / \mathrm{mmnp} / 20105304$

\title{
Modeling the Cancer Stem Cell Hypothesis
}

\author{
C. Calmelet $^{1}{ }^{*}$, A. Prokop ${ }^{2}$, J. Mensah ${ }^{3}$, L. J. McCawley ${ }^{4}$ and P. S. Crooke ${ }^{5}$ \\ ${ }^{1}$ Department of Mathematics and Statistics, California State University Chico \\ ${ }^{2}$ Department of Chemical Engineering, Vanderbilt University \\ ${ }^{3}$ Department of Chemistry, Tennessee State University \\ ${ }^{4}$ Department of Cancer Biology, Vanderbilt University \\ ${ }^{5}$ Department of Mathematics, Vanderbilt University
}

\begin{abstract}
Solid tumors and hematological cancers contain small population of tumor cells that are believed to play a critical role in the development and progression of the disease. These cells, named Cancer Stem Cells (CSCs), have been found in leukemia, myeloma, breast, prostate, pancreas, colon, brain and lung cancers. It is also thought that CSCs drive the metastatic spread of cancer. The CSC compartment features a specific and phenotypically defined cell population characterized with self-renewal (through mutations), quiescence or slow cycling, overexpression of anti-apoptotic proteins, multidrug resistance and impaired differentiation. CSCs show resistance to a number of conventional therapies, and it is believed that this explains why it is difficult to completely eradicate the disease and why recurrence is an ever-present threat. A hierarchical phenomenological model is proposed based on eight compartments following the stem cell lineage at the normal and cancer cell levels. As an empirical test, the tumor grading and progression, typically collected in the pathologic lab, is used to correlate the outcome of this model with the tumor development stages. In addition, the model is able to quantitatively account for the temporal development of the population of observed cell types. Two types of therapeutic treatment models are considered, with dose-density chemotherapy (a pulsatile scenario) as well as continuous, metronomic delivery. The drug hit is considered at the stem cell progenitor and early differentiated specialized cell levels for both normal and cancer cells, while the quiescent stem cell and fully differentiated compartments are considered favorable outcome for cancer treatment. Circulating progenitors are neglected in this analysis. The model provides a number of experimentally testable predictions. The relative importance of the cell kill and survival is demonstrated through a deterministic parametric study. The significance of the stem cell compartment is underlined based on this simulation study. This predictive mathematical model for cancer stem cell hypothesis is used
\end{abstract}

\footnotetext{
${ }^{*}$ Corresponding author. E-mail: ccalmelet@ csuchico.edu
} 
to understand tumor responses to chemotherapeutic agents and judge the efficacy.

Key words: Stem cell, mathematical model, hypothesis, cancer therapy.

AMS subject classification: 92C37, 92C50, 34F05.

\section{Introduction}

Disease models serve as a platform to dissect the biochemical mechanisms of normal phenotypes and the processes which go awry during disease conditions. Particularly in complex, multigenic diseases, molecular studies lead to a greater understanding of the disease, and perhaps more targeted approaches for therapies. Stem cells of both the normal and tumorigenic cell type provide an ideal in silico system in which to study events related to development at the molecular and cellular level. The cancer stem cell hypothesis is an evolving concept of oncogenesis that has recently gained wide acceptance. In its simplest form this hypothesis suggests that many if not all tumors arise by consecutive genetic changes in a small subpopulation of cells termed cancer stem cells (CSCs). The CSCs in turn maintain the tumor in a similar manner as normal stem cells can maintain cell populations within normal organ environments. Molecular biological methods have shed light on the tumorigenic role of CSCs including the activation of the proliferative capacity of quiescent CSCs and their differentiation into mature cell lineages, see Fang et al. [6]. Complementary mathematical algorithms have provided additional alternative insights into underlying biological mechanisms of CSC and have explored their promising potential as novel molecular target. CSCs are considered an intriguing new therapeutic target partly due to the mixed success of current therapeutic regimen that targets mostly the rapidly proliferating and differentiated tumor cells, leaving a remnant of undetectable CSCs that can reform the tumor. The prevailing assumption is that targeting quiescent CSCs with novel therapeutic approaches is a highly viable option for the successful eradication of tumors. The model assumes that initially the tumor is generated by stem cells that have mutated and therefore proliferated and differentiated with deregulated mechanisms.

A cancer stem cell hypothesis postulates that a cell can be reprogrammed to regain properties associated with cancer cells. This concept suggests a view of carcinogenesis not as vague transformation but as well known differentiation. In disease free tissue, a stem cell is defined by unlimited self-renewal and the ability to give rise to one or more highly differentiated cell types. These normal stem cells give rise to progenitor cells, sometimes termed transit amplifying cells that can quickly expand the cell population, see chart in Figure 1. These progenitor cells differentiate into specialized cells that initially retain some ability for cellular division; however, as these cells progress towards terminal differentiation they no longer retain the ability to proliferate. Although CSCs share various characteristics with normal stem cells, including self-renewal, asymmetric cell division, indefinite proliferative capacity, and self-protection mechanisms, they also contain unique and disease-specific features suitable for exploitation as therapeutic targets, see chart in Figure 2. Several existing anticancer agents and experimental therapeutics can inhibit pathways 
critical to CSC maintenance, and could, therefore, be utilized to eradicate CSCs, see Schatton et al. [22]. Therapies targeted at eradicating CSCs may be promising, but their further development will require a more thorough understanding of the biology of CSCs and methods for identifying and isolating this cell subpopulation. Therefore under CSC hypothesis one can naturally conclude that epigenetic alterations and mutations of genes responsible for signal transmission may promote the formation of cancer stem cells. Focusing on the cross-talk between stem cells and their microenvironment is also a promising way to explore how to better target cancer stem cells and be curative. The role of CSCs in multistage cancer progression, particularly with respect to metastasis, has not been well-defined. Mathematical modeling may help to delineate their role by putting forward hypotheses with subsequent verification by experiments.

Cancer is a result of multistage process. Cancerous growth forms as the result of a sequence of events over a period of time. The events are associated with the exposure of DNA to damaging molecules originating from within or outside of the body. Researchers suggest that several physical/chemical insults to susceptible genes will lead to cancer. Cancer arises by the sequential acquisition of mutations leading to the progressive erosion of normal cellular control mechanisms, via accumulation of genetic and epigenetic alterations. In addition, the cellular differentiation should be added to the cancer platform model. Differentiation can act as a fail-safe mechanism against unrestrained cellular growth - much like cell death. In early stages of tumor progression, the benign tumor retains many of the terminally differentiated features of its tissue of origin. Over time, the tumor progresses towards an invasive phenotype with cancer cells that have properties more characteristic of their undifferentiated cellular counterparts. The above theory of cumulative tumorigenic mutations events (including both induction of over-expression and gene silencing) could be in line with the existence of stem cells since the target of such mutations are indeed the cells that are few in numbers and residing in tumors long-term. Thus, our assumption of multistage mutagenesis is a logical explanation if these few cells are CSCs themselves. Alternatively, as stem cells, themselves, already have cellular behaviors such as the ability to invade and migrate to various tissues that are favorable characteristics of cancerous cells, it has also been proposed that stem cells would require fewer genetic and phenotypic alterations to generate a cancer phenotype. As such, this approach represents a paradigm shift. Our paper targets such cells. Prior mathematical models on CSC including that of Enderling et al. (2007) [5], Michor et al (2005) [17] have examined, separately, quantitative aspects of CSCs in hematopoietic cell differentiation with kinetic algorithms. Roeder et al. (2006) [21] and Dingli et al. (2007) [3] have applied different mathematical criteria to model CSC in Chronic Myeloid Leukemia that have provided additional insight into their therapeutic potential. Although significant differences exist in the models, their common limitation lies in their operational specificity to hematological malignancies. We have developed a computational model of CSC that has general applicability to solid tumors including those of the prostate, colorectal, breast and lung.

Marchal et al. (2006) [16] stated that differentiation is a complex multistep process of cell specialization that begins with the installation of a genetic program, specific for a cell lineage. Development of the differentiation program includes the cell-type specific silencing of some genes and the expression of other genes, that regulate the biological functions associated with the cellular type and that distinguish the specialized cells. Terminal differentiation is the end stage of 
this process where the cells irreversibly lose their proliferative capacity and which represents a form of negative control of growing. Regulating molecules interact to produce the correct balance between cellular multiplication and differentiation during embryogenesis and the normal behavior of an adult. Cancer is a process in which changes in regulating circuits are produced, such as proliferation control, the balance between cellular survival and programmed cellular death (apoptosis), the communication with neighboring cells and with the extracellular matrix, angiogenesis, and finally, the migration of the tumor cell, resulting in tissue invasion and metastatic dissemination. This process implies the progressive development of a more malignant phenotype with an increase of genetic alterations involving genes at several levels of expression during long periods of time. These genetic changes uncouple the normal balance between multiplication and cellular differentiation with an increase in the rate of proliferating cells. Classic chemotherapeutical agents have been very important; nevertheless, as the mechanism of action of these drugs depends on the destruction of the neoplastic cells, their beneficial effects are normally accompanied by a notable morbidity, cytotoxicity and long-term use leading to multidrug resistance. The knowledge of the mechanisms involved in differentiation and malignant transformation has allowed the search of alternative routes for anti-tumoral therapy that does not imply cellular death. Differentiation therapy focuses on the development and use of specific agents designed to selectively attract the terminal differentiation process, making the elimination of tumor cells feasible together with the establishment of normal cellular homeostasis. Differentiation therapy is based on the tacit assumption that many neoplastic cell types exhibit reversible defects in differentiation, which upon appropriate treatment, results in tumor reprogramming and a concomitant loss in proliferative capacity and induction of terminal differentiation or apoptosis (programmed cell death), see Leszczyniecka et al. [14].

Next, we review the role of feedback in cell biology and tumor progression. The inclusion of feedback in models is a necessity as without it the proliferation/growth would continue unabated. Additionally, the process is highly tuned to maintain stable cell populations during physiological tissue turnover or to adjust to changes in the system such as when wound repair triggers rapid adjustments in the cell population. General consideration of feedback role in biology was put forward by Pardee (2006) [19] and [20]. As result of extensive experimentation, we are now provided with a plethora of regulatory mechanisms exerted at many levels. Prominent are covalent protein modifications, non-covalent feedback inhibition that modifies enzyme activity, and enzyme induction. Freeman and Gurdon (2002) [7] pointed out that different regulatory strategies operate for threshold. Threshold signaling is extensively regulated by feedback mechanisms. Thomas (1998) [23] reveals that it is now customary to draw a line coupling cell differentiation to the physical concept of multistationarity and of positive feedback, as minimal requirements for differentiation and homeostatic regulation. It has become evident that a complicated interplay between signaling pathways, involving extensive feedback regulation and multiple levels of cross-talk, underlies even the "simplest" developmental decision. Single-negative feedback, double-negative feedback, and positive feedback in biology are very common. Demongeot et al. (2000) [2] employed a concept of regulatory feedback circuitry to oriented cyclic interactions between elements of a system. There are two classes of circuits, positive and negative, whose properties are in striking contrast. Positive circuits are a prerequisite for the occurrence of multiple steady states (multistationarity), 
and hence, they are involved in all processes showing hysteresis or memory. The role of positive circuits in cell differentiation and in immunology is well documented. Negative circuits are involved in homeostatic regulation, with or without oscillations. A simple combination of positive and negative circuits provides powerful regulatory modules, which can also be associated in multistep modules.
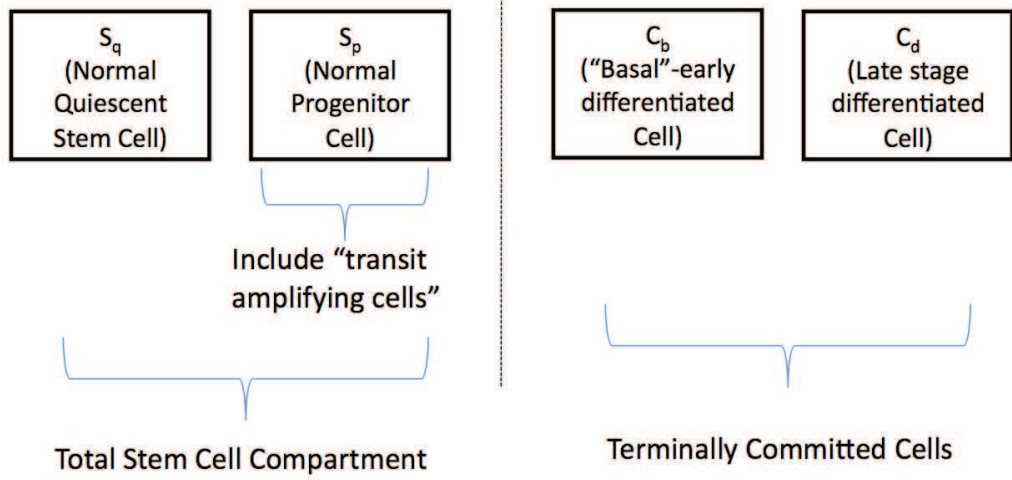

Figure 1: Differentiated and undifferentiated cell compartments

In terms of cancer therapy, one should consider a repeated and continuous dosing (metronomic). Repeated dosing is used with different timing of a high dosage that is for very short periods of time and could be denoted as pulsatile, while the continuous treatment employs low constant dosage of anticancer drugs, often given within the context of combination therapy. The results of recent experimental studies have suggested that frequent administration of certain cytotoxic agents at low doses increases the putative antiangiogenic activity of certain drugs. The advantage of this strategy is lower toxicity and risk of emergence of drug-resistant tumor cells than conventional administration. We use metronomic (MCT) chemo-monotherapy (Laquente et al., 2007) [13]. The metronomic dosing principles are at present poorly defined. Arbitrarily, 10-33\% of the maximum tolerated dose comprises 'the dose range'. We argue that this is too empirical and a better dosing strategy based on a principled approach may help to reduce the problem of empiricism in dosing for metronomic chemotherapy trials.

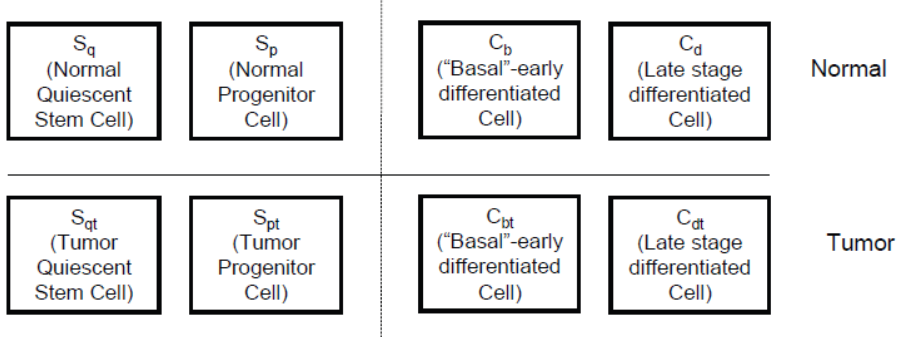

Figure 2: Normal and tumor compartments 


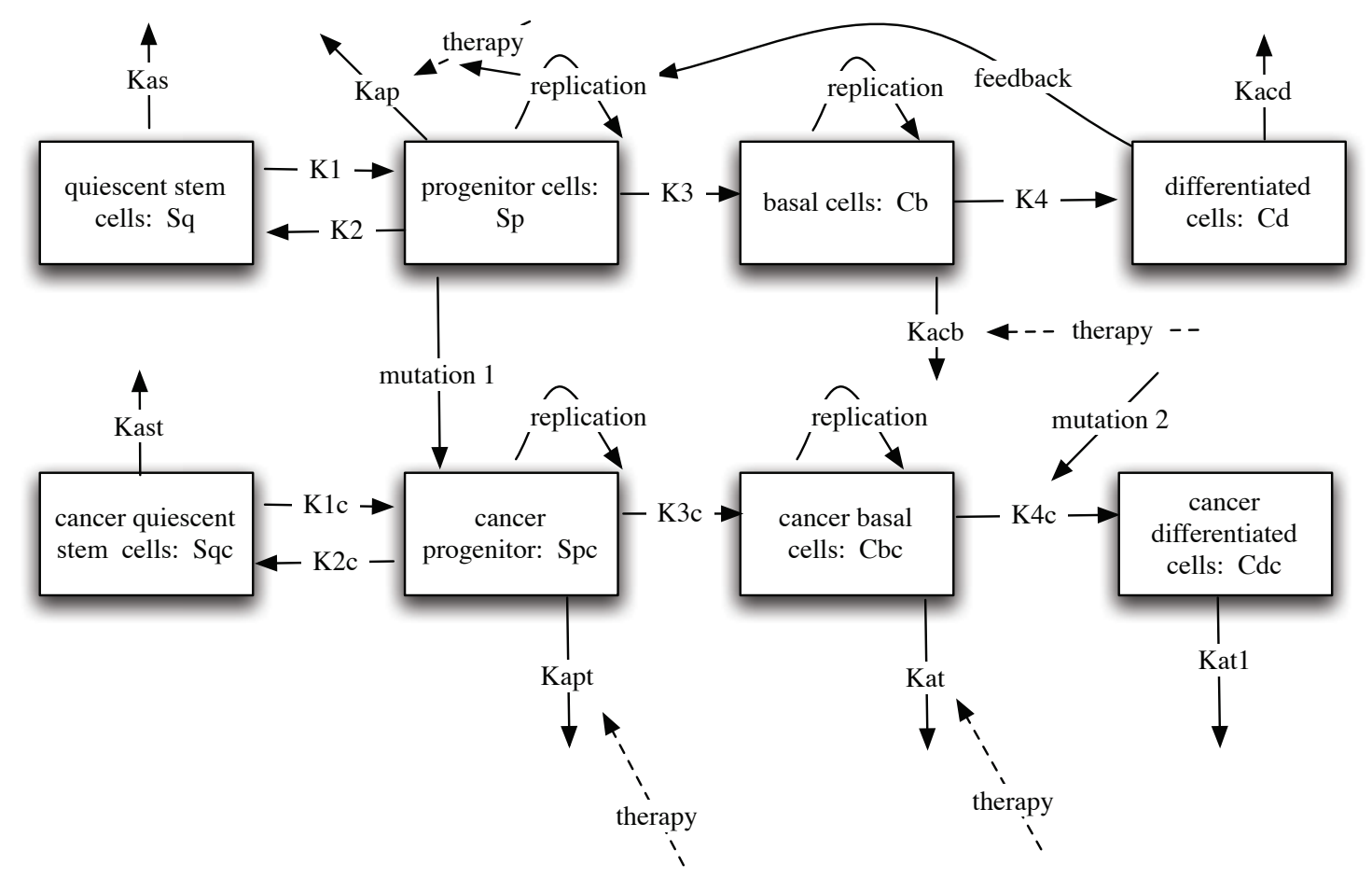

Figure 3: Model compartment diagram

The physiological effects of a drug depend not only on its molecular structure but also on the time-pattern of its administration. We first discuss examples of repeated drug delivery, in standard mode. We then discuss the implications of modeling studies to improve the metronomic drug administration. In all cases, we assume that the differentiated tumors are less responsive to therapy compared to rapidly proliferating partially differentiated tumors. The employment of mathematics allows us to target therapy to any cellular population or compartment of our chosing. In this article, we only explore a monotherapy effects as a combination therapy would be subject of the follow-up paper.

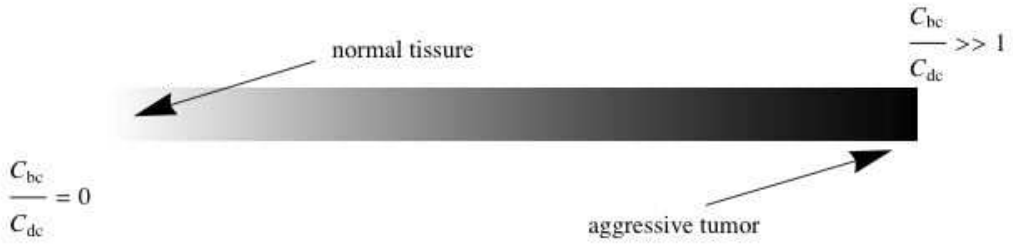

Figure 4: Tissue spectrum 


\section{Mathematical Model}

We study the dynamics in population changes of normal and cancer stem cells and their subsequent progeny. Our model describes the evolution in time of the stem cell population in a domain with no specific geometry. We classified all stem cells into two major groups: the normal and cancer stem cells (see Figure 2). Stem cells have the capabilities to self-renew or differentiate over time into more specialized cells. The total cell population is then classified according to these different stages of their evolution. Cells from each group (normal/cancerous) are divided into four subsequent subgroups or lineages: the quiescent stem cells (inactive), the stem cell progenitors (proliferating, sometimes termed transit amplifying), the basal (early differentiating specialized cells), and the differentiated cells (mature terminally differentiated specialized cells). As the disease progresses into a more malignant stage, the proportion between the number of basal cells and the number of tissue specialized cells increases with time. This proportion called differentiation ratio, is used to distinguish between normal tissue and aggressive tumor. For example, in the case of an invasive tumor, the differentiation mechanism being impaired, produces fewer terminally differentiated cells and therefore yields a higher differentiation ratio. The model consists of eight interconnected compartments regulating the stem cell lineage (see Figure 3). Since we do not consider spatial fluctuations, the description of the model is performed in terms of functions of only one variable: time. We form a system of eight ordinary differential equations for eight functions representing the number of cells per unit volume in each compartment. The system is solved numerically for some given initial conditions and some values of the constant parameters. For each compartment, the variations in time in cell number density are plotted.

We assume that the normal progenitor cells undergo certain mutation hits transforming them into cancerous progenitors, in addition the basal cells have a second mutation hit controlling the differentiation process and transforming a tumor from benign to invasive. In normal population of stem cells, the niche effect is taken into consideration by imposing restrictions on the growth of normal proliferating cells. We assume that the increase in the number of normal progenitors is limited by the number of normal differentiated cells present in the niche. Thus, the normal specialized differentiated cells provide negative feedback to restrict cell proliferation. These conditions no longer hold in disease affected tissue as the tumor grows out of control. Therefore the negative feedback affecting the growth of the cancer stem cell progenitors no longer exists for cancer cells (see Figure 3). Under normal conditions the changes in population size in any of our model compartment depend on the replication rates, death rates, and transfer rates between compartments. These rates depend also on the type of interactions between the different sub-populations of cells assumed in the model.

The model describes the disease progression into different stages from the normal tissue development to the formation of a benign tumor then later to the terminal stage of an invasive tumor. The evolution into different stages of cancer is expressed by the change in the differentiation ratio (see Figure 4). In the case of a benign tumor growth, we presume that this will mimic normal tissue development, and that this ratio decreases in time as the number density of differentiated cells increases during tumor growth. In the case of an invasive tumor the ratio first decreases as it forms a benign tumor growth. The differentiation ratio of the invasive tumor then subsequently 
increases as the tumor progressively matures to an aggressive stage, and can no longer form terminally differentiated cell types. The three main hypotheses consist of the following:

- Malignancy in cancerous stem cells result from mutation hits occurring in the normal stem cell population of progenitors. A mutation rate from the normal progenitor compartment to the cancer progenitor department is introduced. This mutation rate determines the interaction between the normal and the malignant cells and constitutes the first model parameter.

- A second mutation rate is introduced at the cancer-basal cell level to account for the disease progression as the tumors transforms itself from a benign stage to an invasive stage. The differentiation rate is affected by the second mutation reducing over time the amount of differentiated cells.

- We consider the additional effect of a feedback function that limits the growth of the normal stem cell population in function of the number of normal differentiated cells due to the niche effect.

The following nonlinear system of eight equations describes our model (see chart in Figure 4):

$$
\begin{gathered}
\frac{d S_{q}}{d t}=-K_{a s} S_{q}-K_{1} S_{q}+k_{2}\left(S_{p}\right) S_{p} \\
\frac{d S_{p}}{d t}=-K_{a p} S_{p}+\left(\alpha_{p}+\beta\left(C_{d}\right)\right)\left(1-\frac{S_{p}}{S_{p_{\text {max }}}}\right) S_{p}+K_{1} S_{q}-k_{2}\left(S_{p}\right) S_{p}-K_{3} S_{p}-\mu_{1} S_{p} \\
\frac{d C_{b}}{d t}=K_{3} S_{p}-K_{a c b} C_{b}-K_{4} C_{b}+\alpha_{b}\left(1-\frac{C_{b}}{C_{b_{\max }}}\right) C_{b} \\
\frac{d C_{d}}{d t}=K_{4} C_{b}-K_{a c d} C_{d} \\
\frac{d S_{p c}}{d t}=-K_{a s t} S_{q c}-K_{1 c} S_{q c}+k_{2 c}\left(S_{p c}\right) S_{p c} \\
\frac{d C_{b c}}{d t}=K_{p c}+\alpha_{p c}\left(1-\frac{S_{p c}}{S_{p c_{m a x}}}\right) S_{p c}+K_{1 c} S_{q c}-k_{2 c}\left(S_{p c}\right) S_{p c}-K_{b c}-K_{4 c} S_{p c}+\mu_{1} S_{p} \\
\frac{d C_{d c}}{d t}=K_{4 c} C_{b c}-K_{a t 1} C_{d c}
\end{gathered}
$$

This system can be solved numerically for the unknown functions of time $t: S_{p}, S_{q}, C_{b}, C_{d}, S_{p c}$, $S_{q c}, C_{b c}, C_{d c}$ representing respectively the cell number densities of normal progenitor, quiescent, 


\begin{tabular}{|l|c|c|c|c|}
\hline \hline Compartment & R. R. & A. R. & Transfer In & Transfer Out \\
\hline$S_{q}$ - Quiescent & & $K_{a s}$ & $K_{2}$ & $K_{1}$ \\
\hline$S_{p}$ - Progenitor & $\alpha_{p}$ & $K_{a p}$ & $K_{1}$ & $K_{2}, K_{3}, \mu_{1}$ \\
\hline$C_{b}$ - Basal & $\alpha_{b}$ & $K_{a c b}$ & $K_{3}$ & $K_{4}$ \\
\hline$C_{d}$ - Differentiated & & $K_{a c d}$ & $K_{4}$ & \\
\hline$S_{q c}$ - Cancer (Quiescent) & & $K_{a s t}$ & $K_{2 c}$ & $K_{1 c}$ \\
\hline$S_{p c}$ - Cancer (Progenitors) & $\alpha_{p c}$ & $K_{a p t}$ & $K_{1 c}, \mu_{1}$ & $K_{2 c}, K_{3 c}$ \\
\hline$C_{b c}$ - Cancer (Basal) & $\alpha_{b c}$ & $K_{a t}$ & $K_{3 c}$ & $K_{4 c}$ \\
\hline$C_{d c}$ - Cancer (Differentiated) & & $K_{a t}$ & $K_{4 c}$ & \\
\hline
\end{tabular}

Table 1: Kinetic Parameters for Compartments. (R.R.=Replication Rates, A. R.=Apoptotic Rates).

basal, differentiated cells, and its cancerous counterparts. The model parameters are given in Table 1. The constants $S_{p_{\max }}, C_{b_{\max }}, S_{p c_{\max }}, C_{b c_{\max }}$ denote the maximum number of proliferating cells and basal cells for normal and cancer types.

The feedback function is expressed by the composite function that depends on the number of differentiated cells $C_{d}$ and on the coefficient $K_{z}$ (see Table 2) limiting the size of the differentiating cells:

$$
\beta\left(C_{d}\right)=\beta_{0}\left(1-\frac{C_{d}}{C_{d}+K_{z}}\right)
$$

where $\beta_{0}$ is a feedback constant parameter. The rates at which proliferating cells become quiescent for both normal and cancerous cells vary also logistically according to the formulas:

$$
\begin{gathered}
k_{2}\left(S_{p}\right)=K_{2}\left(1-\frac{S_{p}}{S_{p}+S_{p_{\infty}}}\right), \\
k_{2 c}\left(S_{p c}\right)=K_{2 c}\left(1-\frac{S_{p c}}{S_{p c}+S_{p c_{\infty}}}\right)
\end{gathered}
$$

where $S_{p_{\infty}}$ and $S_{p c_{\infty}}$ are limiting coefficients preventing uncontrolled transfers without bound from proliferating compartments into quiescent compartments. The constants $K_{2}$ and $K_{2 c}$ are given transfer rates (see Table 1) representing the rate at which the normal and cancer progenitor cells become quiescent. Considering that natural biological events experience random fluctuations, we assume that the mutation rate $\mu_{1}$ oscillates randomly according to the following formula:

$$
\mu_{1}=\mu_{0}\left(1+r_{0}\right)
$$

where $\mu_{0}$ is a constant parameter and $r_{0}$ is a random number between 0 and 1 . To account for the random variations of the proliferation rates for both normal and cancer cells, we introduce another random parameter $r_{\alpha}$ set between -0.005 and 0.001 that satisfies both equations:

$$
\alpha=\alpha_{0}\left(1+r_{\alpha}\right)
$$



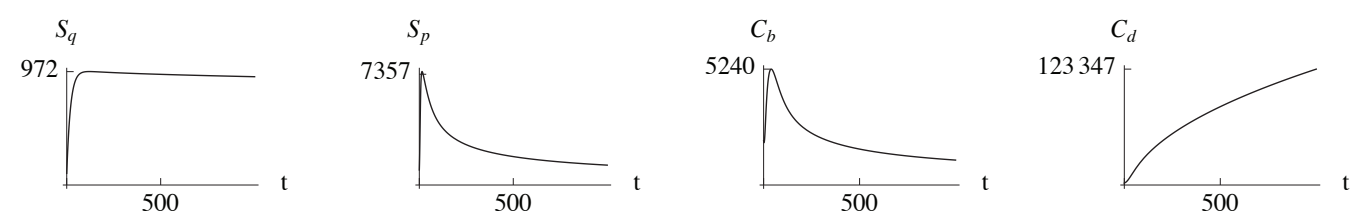

Figure 5: No tumor. $\mu_{1}=.05, \beta_{0}=1$. Initial conditions: $S_{q c_{0}}=S_{p c_{0}}=C_{b c_{0}}=C_{d c_{0}}=0$.

$$
\alpha_{c}=\alpha_{0 c}\left(1+r_{\alpha}\right)
$$

where $\alpha=\alpha_{p}, \alpha_{b}$, and $\alpha_{c}=\alpha_{p c}, \alpha_{b c}$. The second mutation acts on the population of cancer basal cells. As the tumor changes from a benign to a metastatic form by spreading to other locations, the cancer basal cells loose their ability to differentiate. Therefore the number of basal cells that differentiate or the transfer rate from basal to the differentiated compartment starts decreasing. We model this transfer rate as a multistep function that is randomly decreasing in time by the equation:

$$
K_{4 c}(t)=K_{4 c_{0}}\left(1-\delta \sum_{i=1}^{n_{T}} r_{i}(t)\right)
$$

at any time $t,(i-1) \frac{T}{n_{T}} \leq t \leq i \frac{T}{n_{T}}$, and for any positive integer, $i \leq n_{T}$, where $n_{T}$ denotes the number of subintervals during the total time period $T, 0 \leq t \leq T$. $K_{4 c_{0}}$ is the initial constant transfer rate, for an original tumor. The coefficient $\delta$ represents the stage of the tumor: $\delta=0$ (benign) and $\delta=1$ (invasive). The numbers $r_{i}(t)$ are random values taken between 0 and $\mu_{2}$, where $\mu_{2}$ is the second mutation rate.

\section{Numerical Analysis}

\subsection{Disease Progression and Model Predictions}

We show how the model predicts the different stages of the disease, as the tumor progresses from benign to invasive stage depending on the model parameter values. The system is solved numerically given some initial conditions and parameters describing the actual stage of the tumor. All constants are defined in Table 1 and their standard values are listed in Table 2. The solutions are then plotted for different model parameter values, see Figures 5-7. Note that these values are selected from empirical observations relevant to tumor grading and are not obtained by experiments. And in the case of no tumor the densities of cancer cells $S_{p c}, S_{q c}, C_{b c}, C_{d c}$ are all equal to zero.

For each stage of the tumor we define the characteristics of the normal and cancer stem cells and compare with the model numerical outcomes.

In the case of no tumor, the tissue is only composed of normal cells. No cancer cells are actively present, see Figure 5. We notice that the initial conditions differ from the values given in Table 2. Normal growth is observed in the system. 


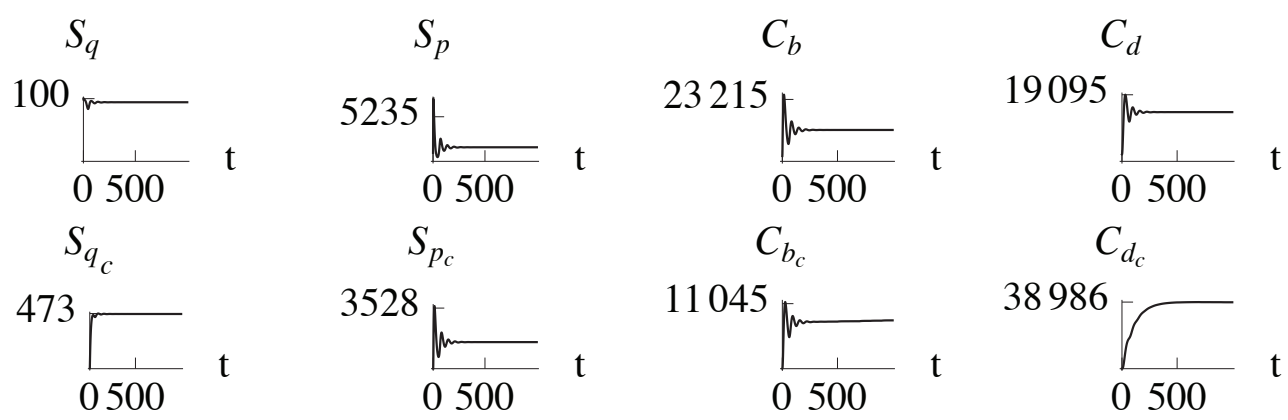

Figure 6: Benign stage tumor. $\mu_{1}=.1, \beta_{0}=5, \mu_{2}=.05, K_{2}=.5, K_{2 c}=.9$.
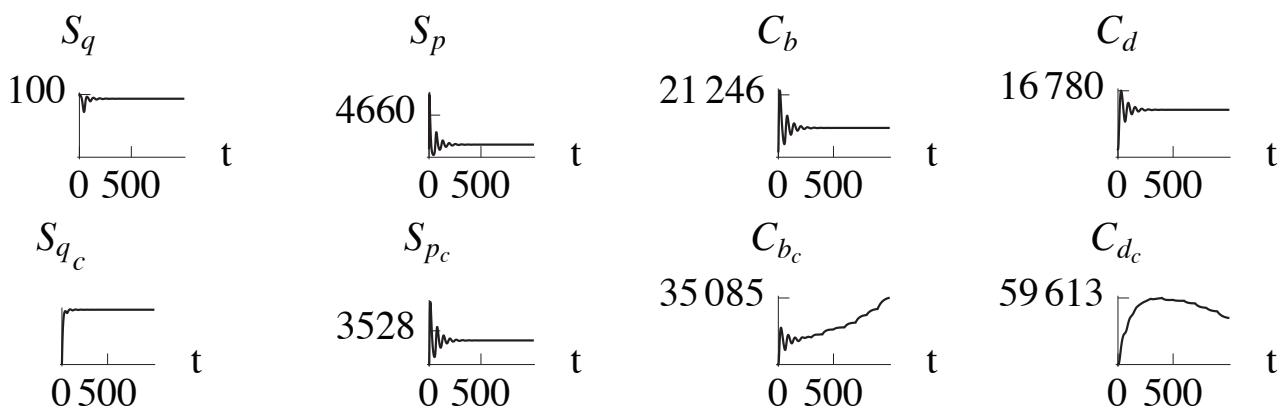

Figure 7: Invasive tumor. $\mu_{1}=.2, \beta_{0}=5, \mu_{2}=.16, K_{2}=.5, K_{2 c}=.2$.

For a benign tumor, the normal stem cell progenitors mutate into malignant cells at a rate $\mu_{1}$. The cancer progenitors differentiate into cancer basal cells which at their turn transform progressively into specialized malignant cells. The number of cells that are terminally differentiated is increasing over time, reaching a steady level that is comparable to normal growth, see Figure 6. Note that in both normal and benign tumor cases, the total stem cell population consisting of quiescent and progenitor populations is 5\%, which is in agreement with experimental values, see [1], [4], [10].

In the invasive case, the tumor can no longer stay confined, becomes metastatic and starts invading the surrounding tissues. The cancer basal cells no longer differentiate effectively into specialized cells. The number of differentiated cells present in the primary tumor starts decreasing over time, and the differentiation rate changes its course from a decreasing to an increasing function of time, see Figure 7.

In addition the model provides a quantitative way to measure the malignancy of a tumor by studying the ratio of the basal cell number density over the differentiated cell number density. Figure 8 shows the plot of the differentiation ratio as well as the plot of the stem cell ratio measuring the number of stem cells (progenitors and quiescent) over the number of non-stem cells (basal and differentiated cells) for a benign tumor. Similarly Figure 9 shows the ratios for an invasive tumor. Comparing the differentiation ratios for benign and invasive tumor, we notice that the ratio is more significant for an invasive tumor. This result is expected since the number of differentiated cells diminishes over time due to the fact that with increasing progression the tumor can no longer form 

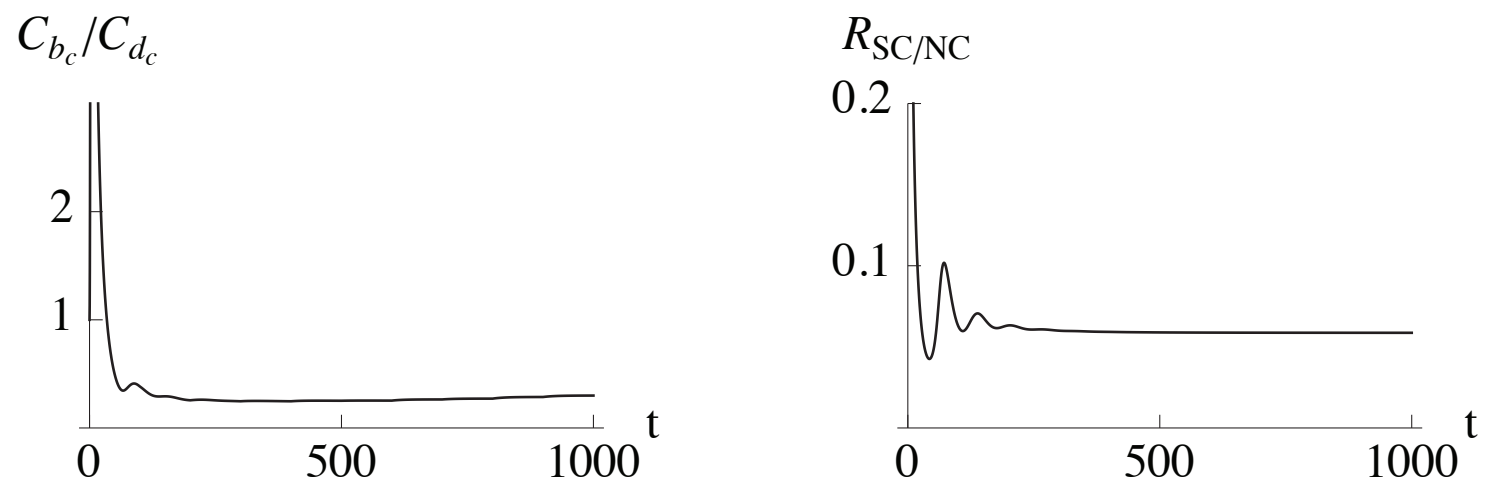

Figure 8: Ratios for Benign Tumor. $C_{b c}$ : Cancer Basal, $C_{d c}$ : Cancer Differentiated, $S C$ : Stem Cells, $N C$ : Non Stem Cells (Basal and differentiated).
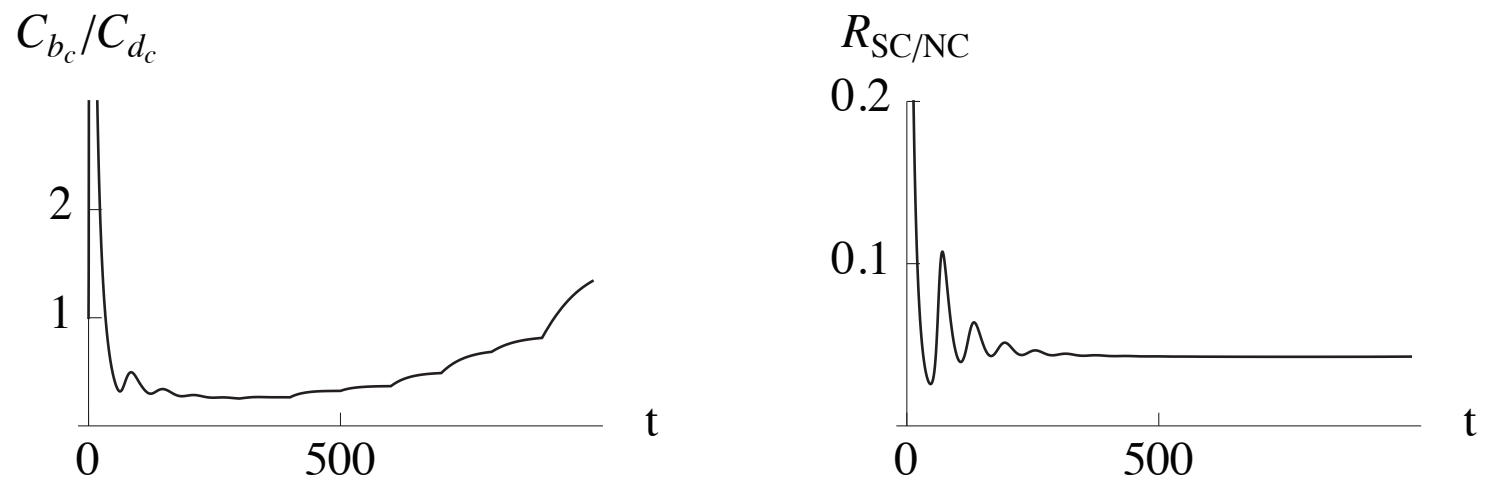

Figure 9: Ratios for invasive tumor. $C_{b c}$ : Cancer basal, $C_{d c}$ : Cancer differentiated, $S C$ : Stem cells, $N C$ : Non stem cells (basal and differentiated). 


\begin{tabular}{|l|c|c|c|c|c|}
\hline \hline I.C. & Max.\& Limits & R.R. & A.R. & In & Out \\
\hline$S_{q_{0}}=100$ & & & $K_{a s}=0.0001$ & $K_{2}=0.05$ & $K_{1}=0.5$ \\
\hline$S_{p_{0}}=1000$ & $\begin{array}{c}S_{p_{\max }}=10000 \\
S_{p_{\infty}}=100\end{array}$ & $\begin{array}{c}\alpha_{p_{0}}=0.1 \\
K_{z}=2000\end{array}$ & $K_{a p}=0.001$ & $K_{1}=0.5$ & $\begin{array}{c}K_{2}=0.5 \\
K_{3}=0.5 \\
0.005 \leq \mu_{1} \leq 0.2\end{array}$ \\
\hline$C_{b_{0}}=2000$ & $C_{b_{\max }}=10000$ & $\alpha b=0.001$ & $K_{a c b}=0.01$ & $K_{3}=0.05$ & $K_{4}=0.06$ \\
\hline$C_{d_{0}}=2000$ & & & $K_{a c d}=0.001$ & $K_{4}=0.06$ & \\
\hline$S_{q c_{0}}=5$ & & & $K_{a s t}=0.001$ & $K_{2 c}=.9, .2$ & $K_{1 c}=0.1$ \\
\hline$S_{p c_{0}}=10$ & $\begin{array}{c}S_{p c_{\max }}=50000 \\
S_{p c_{\infty}}=100\end{array} \alpha_{b c}=0.001$ & $K_{a t}=0.01$ & $K_{3 c}=0.3$ & $\begin{array}{c}K_{4 c_{0}}=0.05 \\
0.01 \leq \mu_{2} \leq 0.2\end{array}$ \\
\hline$C_{b c_{0}}=10$ & $C_{b c_{\max }}=5000$ & $\alpha_{b c}=0.001$ & $K_{a t}=0.01$ & $K_{3 c}=0.3$ & $\begin{array}{c}K_{4 c_{0}}=0.05 \\
0.01 \leq \mu_{2} \leq 0.2\end{array}$ \\
\hline$C_{d c_{0}}=10$ & & & $K_{a t_{1}}=0.01$ & $\begin{array}{c}K_{4 c_{0}}=0.05 \\
0.01 \leq \mu_{2} \leq 0.2\end{array}$ & \\
\hline
\end{tabular}

Table 2: Parameter Values

fully specialized cell types. Secondly, if we compare the stem cell ratios for benign and for invasive tumor, we can see that the decrease is more pronounced in the case of a more advanced tumor.

\subsection{Effect of Therapy}

Unlike conventional therapy that acts on all cells regardless of their type by eliminating at the same time the normal cells, our model can be used for initiating directed therapies with the goal of reducing specific cell populations, in particular the early cancer progenitors and basal cells. The reduction in cell population is considered in the model by assuming an increase in death rate. In other words, treatment application is translated into the model by applying an increase in the apoptotic rate of each cell population that is targeted by therapy. Unlike in the previous section the apoptotic rates are no longer constants but are functions of time. Different treatment procedures have been applied ranging from pulse modulation to continuous therapy. We discuss the effectiveness of each type of treatment. Considering that this effectiveness can be measured by calculating the cancer fraction or ratio between cancer cells and all cells. We therefore assume that for any kind of therapy, the apoptotic rates are no longer constants but increase in time either in a discrete or continuous way.

\subsubsection{Pulse Therapy}

The model assumes a pulsed therapy corresponding to the application of a constant dosage $\Gamma_{P}$ given periodically at different time intervals and alternating with a rest period between each application. The treatment period also increases in duration with time. The analysis is performed for two different time periods $T_{1}(=200)$ and $T_{2}(=500)$ over a total period $T(=2000)$. We consider that the feedback function will also influence the death rates of the normal progenitors. Therefore the apoptotic rate of progenitors depends on the number of proliferating cells and on the number of differentiating cells present in the system according to the following formula:

$$
k_{a p}=\left\{\begin{array}{c}
K_{a p_{0}} \Gamma_{P}, \quad 0 \leq t \leq S_{1}, \quad S_{1}+T_{1} \leq t \leq S_{2}, \quad S_{2}+T_{2} \leq t \leq T \\
K_{a p_{0}} \Gamma_{P}\left[1+\delta_{a p}\left(\alpha_{p}+\beta\left(C_{d}\right)\right)\left(1-\frac{S_{p}}{S_{p_{\max }}}\right)\right], S_{1} \leq t \leq S_{1}+T_{1}, S_{2} \leq t \leq S_{2}+T_{2}
\end{array}\right.
$$


where the first pulse occurs during the first time period, $\left[S_{1}, S_{1}+T_{1}\right]$ and the second pulse, $\left[S_{2}, S_{2}+\right.$ $T_{2}$, where $S_{1}(=500)$ and $S_{2}(=1000)$ are the starting times of the first and second pulse treatment. The constant $\delta_{a p}$ represents the dosage variation coefficient of the therapy. The function $k_{a p}=$ $k_{a p}\left(t, C_{d}, S_{p}\right)$ replaces the constant $K_{a p}$ in the original system of equations.

Similarly the apoptotic rates for normal basal cells, cancer progenitors and cancer basal cells vary in time according to the following:

$$
\begin{gathered}
k_{a c b}(t)=\left\{\begin{array}{c}
K_{a c b_{0}} \Gamma_{P}, \quad 0 \leq t \leq S_{1}, \quad S_{1}+T_{1} \leq t \leq S_{2}, \quad S_{2}+T_{2} \leq t \leq T \\
4 K_{a c b_{0}} \Gamma_{P}, S_{1} \leq t \leq S_{1}+T_{1}, S_{2} \leq t \leq S_{2}+T_{2}
\end{array}\right. \\
k_{\text {apt }}(t)=\left\{\begin{array}{c}
K_{a p t_{0}} \Gamma_{P}, \quad 0 \leq t \leq S_{1}, \quad S_{1}+T_{1} \leq t \leq S_{2}, \quad S_{2}+T_{2} \leq t \leq T \\
4 K_{a p t_{0}} \Gamma_{P}, S_{1} \leq t \leq S_{1}+T_{1}, S_{2} \leq t \leq S_{2}+T_{2}
\end{array}\right. \\
k_{a t}(t)=\left\{\begin{array}{c}
K_{a t_{0}} \Gamma_{P}, \quad 0 \leq t \leq S_{1}, \quad S_{1}+T_{1} \leq t \leq S_{2}, \quad S_{2}+T_{2} \leq t \leq T \\
4 K_{a t_{0}} \Gamma_{P}, S_{1} \leq t \leq S_{1}+T_{1}, S_{2} \leq t \leq S_{2}+T_{2} .
\end{array}\right.
\end{gathered}
$$

Substituting the constant apoptotic rates $K_{a p}, K_{a c b}, K_{a p t}, K_{a t}$, by the new functions of time $k_{a p}\left(t, C_{d}, S_{p}\right), k_{a c b}(t), k_{a p t}(t), k_{a t}(t)$, into the original system and using the values of the parameters given in Table 3, the numerical solutions of the system are plotted considering two cases, see Figures 10-12: First assuming the presence of quiescent cancer stem cells, see Figures 10-12, and secondly assuming their non-existence (without CSCH), see Figures 14-16.

\begin{tabular}{|l|c|c|c|c|c|}
\hline \hline I.C. & Max. \& Limits & R.R. & A.R. & In & Out \\
\hline$S_{q_{0}}=100$ & & & $K_{a s}=0.001$ & $K_{2}=5$ & $K_{1}=1$ \\
\hline$S_{p_{0}}=1000$ & $\begin{array}{c}S_{p_{\max }}=50000 \\
S_{p_{\infty}}=10000\end{array}$ & $\begin{array}{c}\alpha_{p_{0}}=0.01 \\
K_{z}=2000\end{array}$ & $K_{a p 0}=0.1$ & $K_{1}=1$ & $\begin{array}{c}K_{2}=5 \\
K_{3}=0.5 \\
\mu_{1}=0.09\end{array}$ \\
\hline$C_{b_{0}}=2000$ & $C_{b_{\max }}=50000$ & $\alpha b=0.001$ & $K_{a c b 0}=0.02$ & $K_{3}=0.5$ & $K_{4}=0.06$ \\
\hline$C_{d_{0}}=10000$ & & & $K_{a c d}=0.05$ & $K_{4}=0.06$ & \\
\hline$S_{q c_{0}}=100$ & & $K_{a s t}=0.001$ & $K_{2 c}=30$ & $K_{1 c}=0.1$ \\
\hline$S_{p c_{0}}=10$ & $\begin{array}{c}S_{p c_{\max }}=50000 \\
S_{p c_{\infty}}=10000\end{array}$ & $\alpha_{p c 0}=0.02$ & $K_{a p t 0}=0.002$ & $K_{1 c}=2$ & $\begin{array}{c}K_{4 c_{0}}=0.05 \\
\mu_{2}=0.001\end{array}$ \\
\hline$C_{b c_{0}}=10$ & $C_{b c_{\max }}=50000$ & $\alpha_{b c}=0.001$ & $K_{a t 0}=0.02$ & $K_{3 c}=0.3$ & $\begin{array}{c}K_{4 c_{0}}=0.025 \\
\mu_{2}=.001\end{array}$ \\
\hline$C_{d c_{0}}=5$ & & & $K_{a t_{1}}=0.01$ & $\begin{array}{c}K_{4 c_{0}}=0.025 \\
\mu_{2}=.001\end{array}$ & \\
\hline
\end{tabular}

Table 3: Therapy Parameter Values

We examine the different plots obtained in Figures 10-13. In Figure 10 we observe that at each treatment application when death rates are higher, the stem cells increase in number density while the non-stem cell population decreases in number density as time varies. The decrease in number of cancerous tumor cells is significant and shows that pulse therapy is an effective way to treat cancer. It is also noticed that at the second pulse application where treatment lasts for a longer period of time, the number density of cancer differentiated cells reduces even more significantly. Figure 11 shows similar results than Figure 10 with the exception that the quiescent stem cell number density is zero since the quiescent stem cells are assumed inexistent. Comparing results from both cases we observe a slight but noticeable difference. In the next paragraph we consider the continuous therapy and compare its efficacy with pulse therapy. 

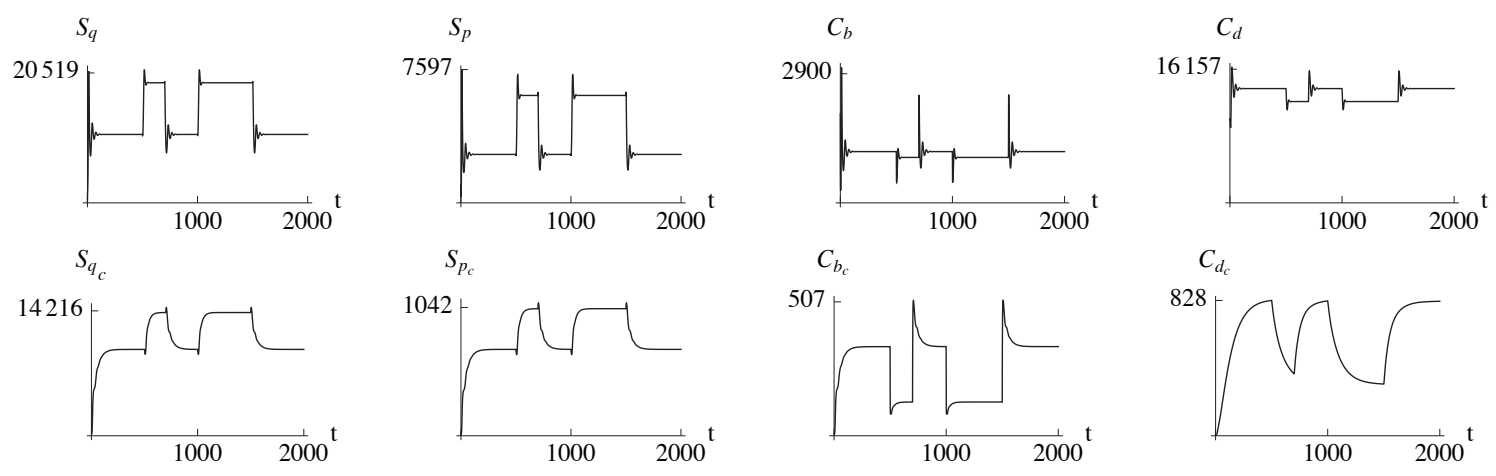

Figure 10: Pulse therapy with CSCH. $\Gamma_{P}=30, T=2000$.
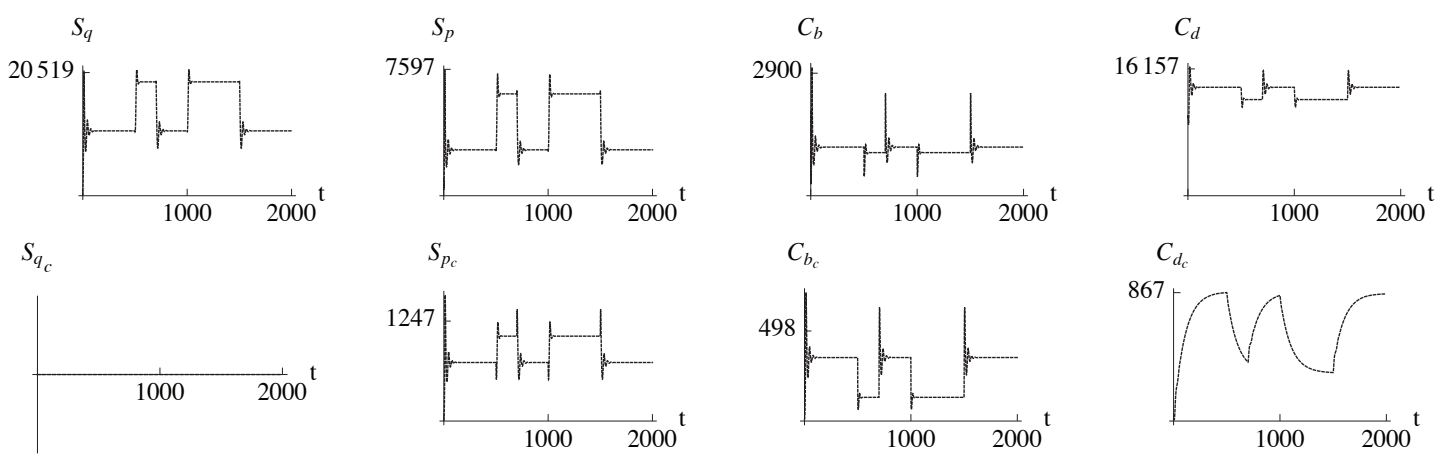

Figure 11: Pulse therapy without CSCH. $\Gamma_{P}=30, T=2000$.
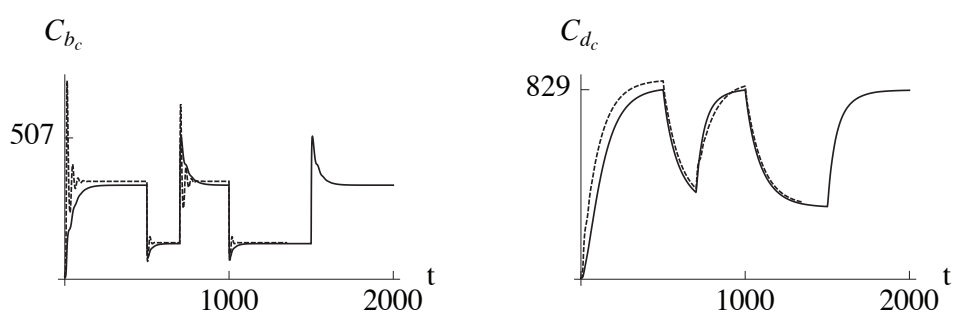

Figure 12: Cancer basal cells , cancer differentiated cells with pulse therapy. Plain=with quiescent cancer stem cells (QCSC), dotted $=$ without QCSC. $\Gamma_{P}=30, T=2000$. 


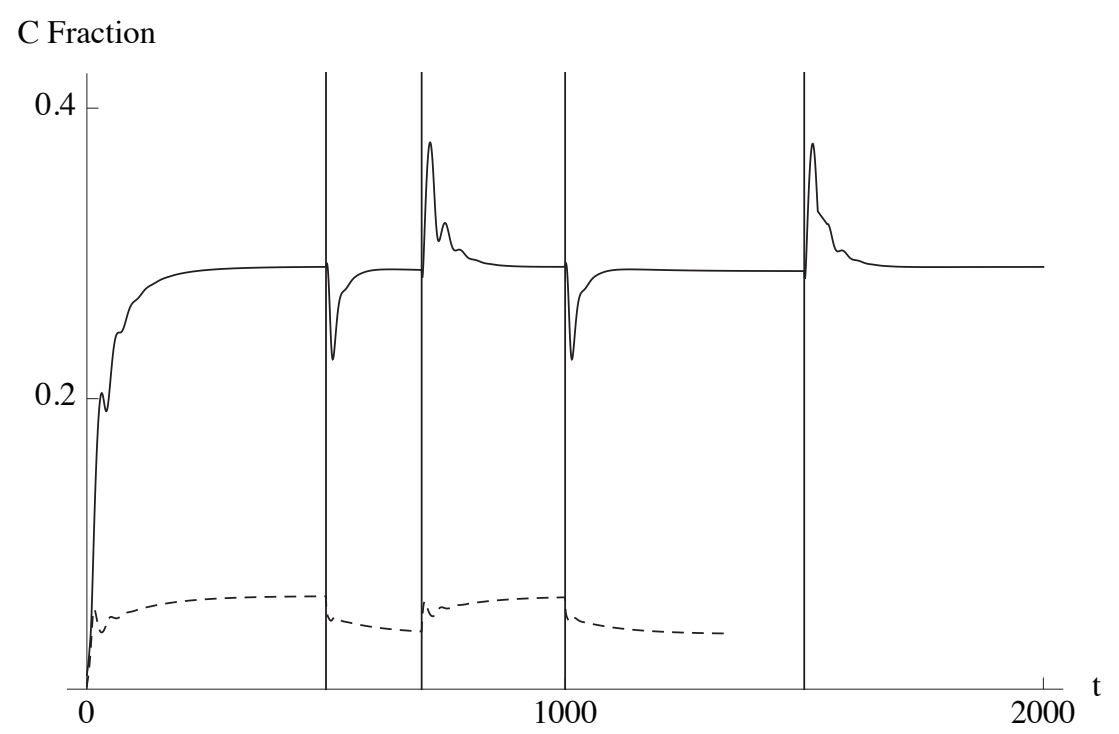

Figure 13: Cancer ratio for pulse therapy. Plain=with quiescent cancer stem cells (QCSC), dotted =without QCSC. $\Gamma_{P}=30, T=2000$.

\subsubsection{Continuous Therapy}

We consider that therapy is given continuously at low dosage. We repeat a similar analysis where pulse dosage $\Gamma_{P}(=30)$ is replaced by continuous dosage $\Gamma_{C}(=10)$ over a time period $T(=2000)$. Unlike pulse therapy there is no rest period during treatment. The apoptotic rates for normal progenitor cells are no longer multistep functions but satisfy the equations:

$$
k_{a p}\left(t, C_{d}, S_{p}\right)=K_{a p_{0}} \Gamma_{C}\left[1+\delta_{a p}\left(\alpha_{p}+\beta\left(C_{d}\right)\right)\left(1-\frac{S_{p}}{S_{p_{\max }}}\right)\right] .
$$

For the remaining cells, the rates are assumed to be constants of the form:

$$
\left(k_{a c b}, k_{a p t}, k_{a t}\right)=\left(K_{a c b_{0}}, K_{a p t_{0}}, K_{a t_{0}}\right) \Gamma_{C}
$$

Note that only the normal progenitor death rate varies in time depending on the feedback function. Substituting the apoptotic rates into the model equations we calculate and plot all cell number densities. We also distinguish two cases assuming the existence and non-existence of the quiescent stem cells.

We observe a decrease in the number of cancer cells after application of the pulse therapy. The pulse therapy seems to reach better results than the continuous therapy.

Figure 14 and Figure 15 show that all cell number densities reach a steady state level. Looking at the terminally differentiated cells, unlike pulse therapy there is no decrease in cell number density. It is further noticed that even if the continuous dosage is assumed to have similar value than in the pulsatile case, all cell number densities still increase reaching a steady state level. Therefore we can say that the decrease in number density of cancer cells which was observed after pulse therapy is not replicated in the case of continuous treatment even for equal amount of dosage. 

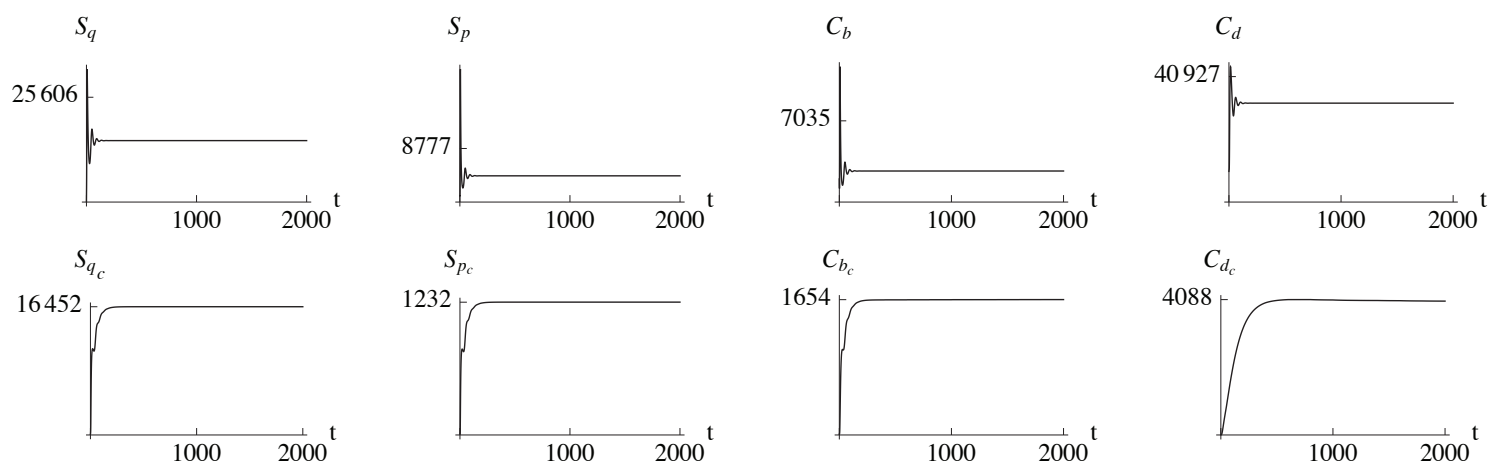

Figure 14: Continuous therapy with CSCH. $\Gamma_{C}=10, T=2000$.
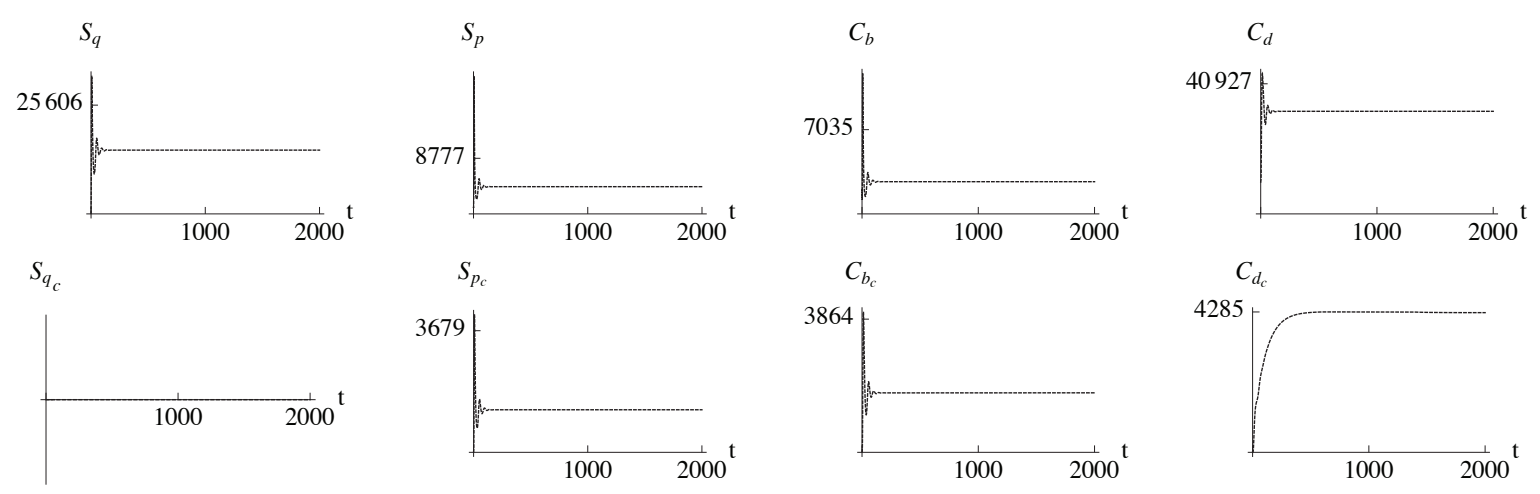

Figure 15: Continuous therapy without $\mathrm{CSCH} . \Gamma_{C}=10, T=2000$.
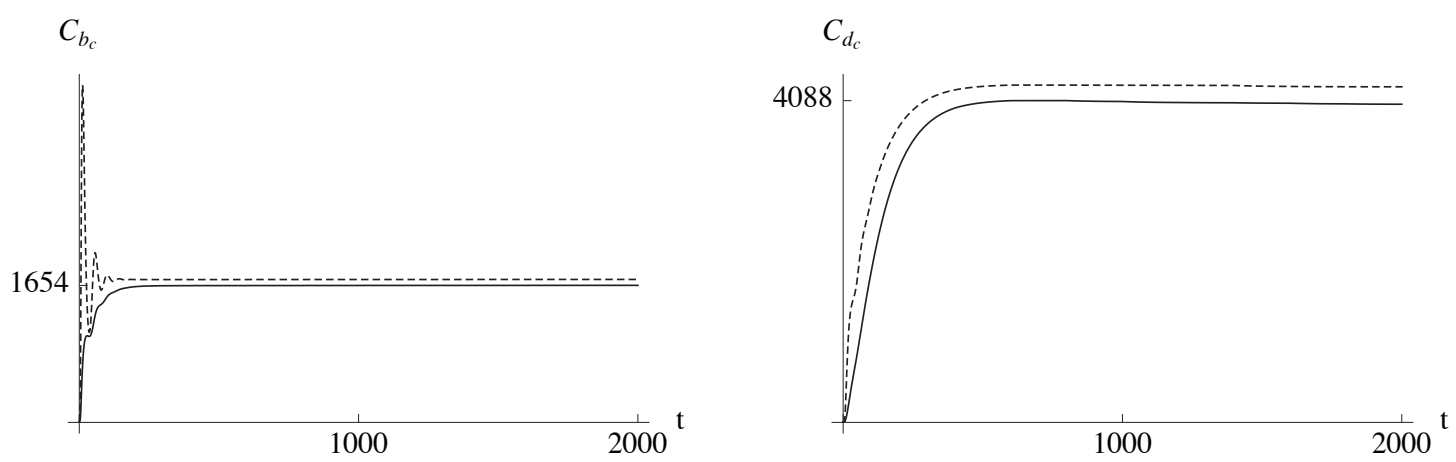

Figure 16: Cancer basal cells, cancer differentiated cells with continuous therapy. Plain=with $\mathrm{CSCH}$, dotted $=$ without $\mathrm{CSCH} . \Gamma_{C}=10, T=2000$. 


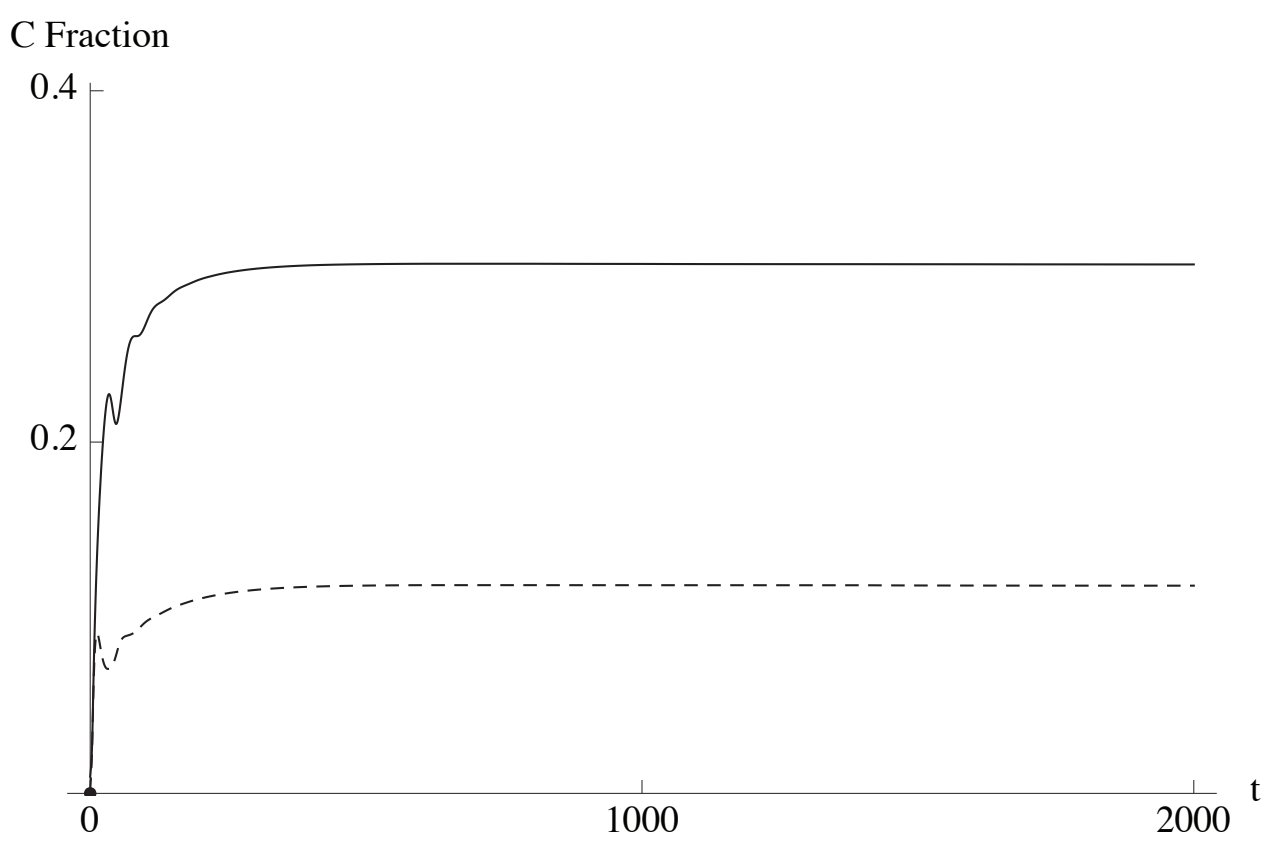

Figure 17: Cancer ratio for continuous therapy. Plain=with QCSC, dotted =without QCSC. $\Gamma_{C}=$ $10, T=2000$.

From the plots of C-fraction, see Figures 13 and 17, we study the proportion of cancer cell number densities versus all cell number densities of the system after therapy. We observed a decrease after application of pulse treatment, however this decrease is not observed in the case of continuous therapy since the C-fraction increases to a steady state level.

In overall, we note that pulse therapy seems to reach better results than continuous therapy. In other words continuous therapy is not as effective.

If we study the significance of the quiescent stem cells and compare the plots for the cases of existence and non-existence of the quiescent cancer stem cells, similar to the pulse therapy there is a small difference in the number of malignant tumor cells.

\section{Conclusion}

We formulated a mathematical model describing the time evolution of cells by studying the variations of cell number densities for normal and cancerous cell lineage. We obtained numerical values of cell number density in each cell compartment for different values of cell parameters. Our model is validated comparing the tumor progression phenotypes to numerical results. We considered two types of therapy (pulse and continuous) and compared their effectiveness using numerical outcomes.

The model has several limitations: Only one negative feedback is considered in the original equations. In addition, we modeled anti-tumor therapy treatment that was cytotoxic by enhancing cell death. Therefore therapy is expressed in the model in changes related only to apoptotic rates 
presuming that an efficacious anti-tumor drug therapy would result in cell killing of the targeted cell population. Cytostatic therapies are not explored in this paper. General empirical observations are used to compare with model outcomes. On the other hand, our model offers the following advantages. With little computational efforts the model can be adjusted to examine a broad range of therapeutic effect on CSCs within each of the 8 compartments. This computational ability represents an advantage over existing models and permits the assessment of alterations in the listed stage-specific phenotypic features after pulse and continuous therapy. This model can also distinguish between beneficial effects on tumor cell eradication as well as harmful side-effects damaging the normal un-diseased tissue population. The model can also be adjusted to determine the best therapeutic choice that enhances the apoptotic cell death of CSC and eventual disease eradication.

Feedback is common in cancer systems as well. Defective controls cause diseases such as cancer and neurological malfunctions. The precise mechanism(s) by which Myc targets signaling pathways are largely unresolved, but a present understanding involves crosstalk and feedback regulatory loops between arbiters of cell death (Nilsson 2003) [18]. In modeling, Ganguly \& Puri [8], [9] proposed a predictive mathematical model for the cancer stem cell hypothesis to gain understanding of tumor responses to chemotherapeutic drugs and to judge the efficacy of treatments in arresting tumor growth. The impact of varying drug efficacies on different abnormal cell populations was investigated through the kinetics associated with their decline in response to therapy. The growth of abnormal (stem and early progenitor) cells from their normal counterparts are typically described with separate mutation probabilities. Tindall (2007) [24] employed a model that considered cells to exist in one of two compartments: proliferating and quiescent, as well as accounting for necrosis and apoptosis with some feedbacks. Differentiation model with a mix of positive and negative feedbacks for hematopoietic stem cells was proposed by Johnston et al. (2007) [11] for a single colorectal crypt by using a compartmental approach, which accounts for populations of stem cells, differentiated cells, and transit cells. The mechanism that could regulate is a feedback as the growth of cell numbers and maintenance of the equilibrium that is normally observed in the crypt. King-Smith \& Morley (1970)[12] proposed an existence of two feedback loops, regulating stem cell stage and release of differentiated cells. Wing-Cheong Lo et al. [25] studied the stability of the olfactory neuroepithelium system in the mouse using a negative feeback that autoregulates the regeneration of the terminally differentiated cells. In our modeling effort we employed a single negative feedback.

Mathematical modeling will help our understanding of cancer cell biology via exploring certain hypotheses in silico as successful cancer therapies would require in-depth understanding. It could also help to design a therapy regimen by exploring the cell behavior and their dynamics under a mono or combination therapies (below) and how CSCs could be depleted by targeted or cytotoxic drugs. Initially, a baseline normal stem cell model is proposed featuring populations of different cell categories, their proliferation, serial differentiation or apoptosis, and exhibiting a low stem cell compartment and a negative feedback from differentiated cells to normal progenitor cells. The construction of mathematical models is aimed to guide the design of improved or modified stem cell niche for the desired alternation of cell fate. A multitude of variables, such as cellcell interactions, the cells physiologic properties, and the state of environment, control the stem cell fate. The structure of the feedback loops that maintain cell homeostasis can have a dramatic 
impact on the probability of cancer development. In this model, only one type of feedback is considered, although a multitude of feedbacks, including multiple independent positive loops, have been observed or speculated. The aim is to prevent regrowth of resting tumor cells by a continuous or pulsatile administration.

A cancer level, likewise, assumes quiescent and progenitor stem cells, transitional (breakdown into different stages is neglected) and fully differentiated cancer cells with no feedback control. Two controlled oncogenic mutation events are also introduced: at the level of normal progenitor to cancer progenitor cells, and from cancer basal cells to terminally differentiated cancer cells (in fact disrupting differentiation process). The shedding of restrictions on cell division and survival, if unchecked, ultimately leads to uncontrolled cell proliferation and cancer. The reversibility of events (e.g., from cancer progenitor cells to cancer quiescent cells) could be addressed at the later stages, likewise chemotherapeutic agents that act by promoting differentiation (e.g., ATRA).

The introduction of the "maximum tolerated dose" in usual treatment protocols (and its concomitant overt toxicity) made necessary the imposition of rest periods between cycles of therapy-a practice that not only involves re-growth of tumor cells, but also growth of selected clones resistant to the therapy. To avoid the problems caused by traditional chemotherapeutic regimens, a new modality of drug administration called "metronomic chemotherapy" has been proposed. This name makes reference to the chronic, equally spaced or continuous administration of (generally) low doses of various chemotherapeutic drugs. The novelty of this treatment modality lies not only in its anti-tumor efficacy with very low toxicity. Metronomic chemotherapy would be the rational option instead of conventional high dose (often escalating) chemotherapy. In that mode, drug application is stopped upon attaining a certain high level of toxicity and subsequent resumption is possible once the toxicity drops below a certain low level. Large delays in subsequent drug administration can thus effectively handle toxicity and it may retard the therapeutic potential due to excessive tumor growth in the absence of drug. As the transition of a stem cell from quiescent to a self-renewing, proliferative state is rare, a potential added benefit is the likelihood of targeting a stem cell with cytotoxic therapies that depend upon cells in the active process of cellular division. In addition, a continuous administration of drug that targeted the proliferative progenerator cell population, would presumably extend remission and delay a repopulation of tumor tissue. In our model the application of directed pulse therapies by increasing the death rates of progenitors and early differentiated cells yields a decrease in the number of cancer differentiated cells, in case of continuous therapy the treatment prevents tumor growth since the number of cancer differentiated cells reaches a steady state level. Therefore targeting the progenitor cells delays the progression of the disease and may result in cancer remission. A mathematical model that may act as a general guideline to tackle the trade-off issues in cancer treatment is provided. Rigorous simulation exercises are required to establish the concept of MCT in the backdrop of conventional cancer treatment practices. Mathematical modeling and computer simulation techniques can assist in this regard. This, in turn, may help in the drug application decision. It is particularly important in situations where a fluctuating clearance rate of the applied drug is present. So, from dynamical perspectives, the frequencies and timings of drug stoppages need to be determined appropriately for maintaining the stability of the system without overburdening the toxicity level.

We have constructed a mathematical model to describe the dynamics of normal and cancer 
stem cell populations based on the general characterization of a tumor. Our model describes the different stages in tumor progression for some parameter values. These values are not in direct correlation with experimental data and are not considered for a particular type of cancer. We have considered two types of therapy (pulsatile and continuous) and studied the effect of therapy on cell density distribution for the different cell compartments. We conclude that pulse therapy is more effective than continuous therapy.

Targeting CSC may offer additional therapeutic window for success in disease elimination. And mathematical modeling of CSC may provide insight into the underlying mechanism for disease prevention and for the provision of novel insight into new therapeutic formulations. A more sophisticated model considering multi-step processes with several feedback functions and the consideration of different type of treatment application involving molecular mechanisms for example, would be very beneficial in future studies.

\section{Acknowledgements}

We would like to express our sincere appreciation to D. Hong (Middle Tennessee State University), J. Wang (Emory University), J. Jeon and M. Ciobanu (Vanderbilt University) for their participation in the original discussion of this work, and to the VICBC(Vanderbilt Integrative Cancer Biology Center) members who hosted the conference. This work was supported in part by the National Cancer Institute Integrative Cancer Biology Program (U54 CA113007).

\section{References}

[1] R.W. Cho, X. Wang X, M. Diehn, K. Shedden, GY Ghen, G Sherlock, A Gurney, J Lewicki, MF Clarke. Isolation and molecular characterization of cancer stem cells in MMTV-Wnt-1 murine breast tumors. Stem Cells, 26 (2008), No. 2, 364-71.

[2] J. Demongeot, M. Kaufman, R. Thomas. Positive feedback circuits and memory. C. R. Acad. Sci. III. 323 (2000), No.1, 69-79.

[3] D. Dingli, A. Traulsen, J. Pacheco. Stochastics Dynamics of Hematopoietic Tumor Stem Cells. Cell Cycle 6 (2007), No.4, 461-466.

[4] V.S. Donnenberg, R.J. Landreneau, A.D. Donnenberg. Tumorigenic stem and progenitor cells: implications for the therapeutic index of anti-cancer agents. Journal Control Release, 122 (2007), No. 3, 385-91.

[5] H. Enderling, M. A. Chaplain, A.R. Andersona, J. S. Vaidyab. A mathematical model of breast cancer development, local treatment and recurrence. Journal of Theoretical Biology, 246 (2007) No. 2, 245-259. 
[6] D. Fang, T. K. Nguyen, K. Leishear, R. Finko, A. N. Kulp, S. Hotz, P. A. Van Belle, X. Xu, D. E. Elder, M. Herlyn. A tumorigenic subpopulation with stem cell properties in melanomas. Cancer Res. 65, (2005), No. 20, 9328-37.

[7] M. Freeman, J.B. Gurdon. Regulatory principles of developmental signaling. Annu. Rev. Cell. Dev. Biol. 18 (2002), 515-39.

[8] R. Ganguly, I.K. Puri. Mathematical model for the cancer stem cell hypothesis. Cell Prolif. 39 (2006), No. 1, 3-14.

[9] R. Ganguly, I.K. Puri. Mathematical model for chemotherapeutic drug efficacy in arresting tumour growth based on the cancer stem cell hypothesis. Cell Prolif. 40 (2007), No. 3, 33854.

[10] R.P. Hill. Identifying cancer stem cells in solid tumors: case not proven. Cancer Res. 66 (2006), No. 4,1891-5.

[11] M.D. Johnston, C.M. Edwards, W.F. Bodmer, P.K. Maini, S.J. Chapman. Mathematical modeling of cell population dynamics in the colonic crypt and in colorectal cancer. Proc. Natl. Acad. Sci. 104 (2007), No.10, 4008-13.

[12] E.A. King-Smith, A. Morley. Computer simulation of granulopoiesis: normal and impaired granulopoiesis. Blood. 36 (1970), No.2, 254-62.

[13] B. Laquente, F. Vials, J.R. Germ. Metronomic chemotherapy: an antiangiogenic scheduling. Clin. Transl. Oncol. 9 (2007), No. 2, 93-8.

[14] M. Leszczyniecka, T. Roberts, P. Dent, S. Grant, P.B. Fisher. Differentiation therapy of human cancer: basic science and clinical applications. Pharmacol Ther. 90 (2001) No. 2-3, 105-56.

[15] I. Malanchi, J. Huelsken. Cancer Stem cells: never Wnt away from the niche. Current Opinion in Oncology 21 (2008), 41-46.

[16] J.A. Marchal, F. Rodrguez-Serrano, R. Madeddu, H. Boulaiz, A. Martinez-Amat, E. Carrillo, O. Caba, J.C. Prados, C. Velez, C. Melguizo, A. Montella, A. Aranega. Differentiation: an encouraging approach to anticancer therapy. J. Anat. Embryol. 111 (2006), No.1, 45-64.

[17] F. Michor, T.P. Hughes, Y. Iwasa, S. Branford, N. P. Shah, C. L. Sawyers, M. A. Nowack. Dynamics of Chronic Myeloid Leukaemia., Nature 435 (2005)1267-1270.

[18] J.A. Nilsson, J.L. Cleveland. Myc pathways provoking cell suicide and cancer. Oncogene. 22 (2003), No. 56, 9007-21.

[19] A.B. Pardee. Regulatory molecular biology. Cell Cycle. 5 (2006), No. 8, 846-52.

[20] A.B. Pardee. Tumor progression-targets for differential therapy. J. Cell Physiol. 209 (2006), No. 3, 589-91. 
[21] I. Roeder, M. Horn, I. Glauche, A. Hochlaus, M. C. Mueller, M. Loeffler. Dynamic modeling of imatinib-treated chronic myeloid leukemia: functional insights and clinical implications. Nature Medicine 12 (2006), 1181-1184.

[22] T. Schatton, N.Y. Frank, M.H Frank. Identification and targeting of cancer stem cells. Bioessays 31 (2009) No. 10, 1038-49.

[23] R. Thomas. Laws for the dynamics of regulatory networks. Int. J. Dev. Biol. 42 (1998), No. $3,479-85$.

[24] M.J. Tindall. Modelling the cell cycle and cell movement in multicellular tumour spheroids. Bull. Math. Biol. 69 (2007), No. 4, 1147-65.

[25] W. C. Lo, C. S. Chou, K. K. Gokoffski, F. Y. Wan, A.D. Lander, A. L. Calof, Q. Nie. Feedback regulation in multistage cell lineages. Math. Biosci. Eng. 6 (2009), No. 1, 59-82. 